\title{
Reexamination of experimental tests of the fluctuation theorem
}

\author{
Onuttom Narayan ${ }^{1}$ and Abhishek Dhar ${ }^{1,2}$ \\ ${ }^{1}$ Department of Physics, University of California, \\ Santa Cruz, CA 95064 \\ 2 Raman Research Institute, Bangalore 560080.
}

(Dated: July 10, 2018)

\begin{abstract}
The Fluctuation Theorem and the Jarzynski equality are examined in the light of recent experimental tests. For a particle dragged through a solvent, it is shown that $Q$, the heat exchanged with the reservoir, does not obey the Jarzynski equality due to slowly decaying tails in its distribution. For molecular stretching experiments, substantial corrections to the Jarzynski equality can result from not measuring the force at the end of the molecule that is moved. We also present a proof of the Fluctuation Theorem for Langevin dynamics that is considerably simpler than the standard proof.

PACS numbers: 05.70.Ln, 82.20.Wt
\end{abstract}

\section{INTRODUCTION}

Understanding how entropy behaves is the fundamental issue in statistical mechanics. Equilibrium statistical mechanics provides a powerful framework to examine a broad variety of systems, but is by its nature restricted to systems in equilibrium. Recently, a remarkable Fluctuation Theorem was proved 1] for entropy growth or decay for systems in a non-equilibrium steady state where the microscopic dynamics are time reversal invariant. This theorem states that in a time interval $\tau$, the probability $p(S)$ of an entropy $S$ being generated satisfies the condition

$$
\lim _{\tau \rightarrow \infty} \ln \frac{p(S)}{p(-S)}=S / k_{B}
$$

where $k_{B}$ is Boltzmann's constant. The theorem was motivated by numerical results on a shear stress model [2]. In Eq.(1) entropy is defined in dynamical systems terms, through phase space contraction and expansion, rather than thermodynamically.

When instead of being in a non-equilibrium steady state, the system starts in thermal equilibrium and then subject to a time-independent perturbation, a stronger result can be proved [3]:

$$
\ln \frac{p(S)}{p(-S)}=S / k_{B}
$$

valid irrespective of the length of the observation time interval $\tau$. Eqs.(1) and (2) are referred to as the Steady State Fluctuation Theorem (SSFT) and the Transient Fluctuation Theorem (TFT) respectively. A simple example illustrating the need for the $\tau \rightarrow \infty$ limit in the first but not the second is given in Ref. [4]. The fluctuation theorem was first proved for thermostatted Hamiltonian systems, and later for systems undergoing Langevin dynamics [5]. Apart from numerical realizations [2, 6], experimental tests of the fluctuation theorem have also been performed 7,8 ].

A related result was proved by Jarzynski [9], relating the change in free energy of a system that starts in equi- librium at a temperature $T$, when it is perturbed externally by making its Hamiltonian time dependent. If the Hamiltonian starts and stops changing at time $t=0$ and $t=\tau$ respectively, and $W_{J}$ is the generalized work defined as $\int_{0}^{\tau} d t \partial_{t} H(t)$, then the Jarzynski equality states that

$$
\left\langle\exp \left[-\beta W_{J}\right]\right\rangle=\exp [-\beta \Delta F]
$$

where $\beta=1 / k_{B} T$. The change in the free energy of the system between equilibrium at temperature $T$ with Hamiltonian $H(0)$ and equilibrium at the same temperature with Hamiltonian $H(\tau)$ is denoted by $\Delta F$. The Jarzynski equality relates the change in free energy, an equilibrium concept, to an average of nonequilibrium measurements. It has been used in experiments where a molecule is stretched at a finite rate (i.e. not adiabatically) to obtain the free energy change due to stretching [10]. The connection between the Jarzynski equality and the TFT was shown by Crooks [11] who showed that if an externally driven system starting in thermal equilibrium is compared to the same system with the external driving time-reversed, and there is a quantity $\omega$ that satisfies

$$
\frac{p_{+}(\omega)}{p_{-}(-\omega)}=\exp [\omega]
$$

where the \pm subscript refer to the original and the timereversed process respectively, then

$$
\langle\exp [-\omega]\rangle_{+,-}=1 \text {. }
$$

He then showed that $S / k_{B}$ is expected to satisfy Eq.(4) for $\omega$, and related $\omega$ to $W_{J}$ on thermodynamic grounds yielding Eq.(3). When the original and time-reversed driving are equivalent, as with a steady state perturbation, the subscripts in Eq. (4) can be dropped, and Eq.(2) results.

Despite the dramatic progress in the field, some uncertain points remain. The relationship between the actual physical work done on a system and the generalized work $W_{J}$ can be tricky 12, 13]. Since Crooks' connection [1] 
between the TFT and the Jarzynski equality uses thermodynamic arguments, it deals with the physical work $W$ rather than $W_{J}$. Questions have also been raised about possible alternative definitions of entropy generated [14, 15], and whether the equations above would be satisfied. There are also issues about the experiments, and whether they measure what they should. In these paper, we seek to clarify some of these points.

The organization of the rest of this paper and its main results are as follows. Section II discusses the connection between $W_{J}$ and the physical work $W$ in various different cases, showing that Eq.(3)) (with the free energy appropriately defined) is always obtained. Section III considers an alternative definition of entropy fluctuations 14, 15] in an experimental context [3], and shows that Eq. (51) is not obeyed; the violation of Eq. (5.5) grows worse as the measuring time interval $\tau$ is increased. Section IV considers the molecular stretching experiments [10] and whether they satisfy the conditions required to invoke the Jarzynski equality, demonstrating that in general there are substantial corrections. Finally, Section V presents a very simple proof of the fluctuation theorem for Langevin dynamics [5], generalizes it when the dynamical equations are linear, and discusses extensions of Eq.(31).

\section{PHYSICAL WORK AND GENERALIZED WORK}

Eq.(3) is stated in terms of the generalized work $W_{J}$, and was derived [9] for a system at constant volume, in which case $\Delta F$ is the change in Helmholtz free energy, $\Delta A$. On the other hand, Crooks 11] obtained the same result on thermodynamic grounds, which therefore involves the actual work $W$ done by the external agent that changes the Hamiltonian. As pointed out previously [12, 13], the two are not always equal. For example, if a time dependent force $f(t)$ is applied to a polymer chain, it is equivalent to a time dependent term in the Hamiltonian $-f(t) x$. The work done along a trajectory $\int d t f(t) \dot{x}(t)$ differs from $W_{J}=-\int x d f(t)$ by $f(\tau) x(\tau)-f(0) x(0)$. However, $W_{J}$ is not always different from the physical work. If the same polymer chain is stretched by holding its end at a fixed location and moving this location, $\partial_{t} H=\dot{x} \partial_{x} H$, and the force exerted on the end by the polymer is equal to $-\partial_{x} H$, so that the work done by the external agent in moving the end is equal to $W_{J}$ [16]. We distinguish, therefore, between experiments where the variation of the Hamiltonian can be expressed as the change of a coordinate, and those where it can be expressed as the change of a force. In the first case, there is no difference between the actual work and $W_{J}$ 17]. Since in the latter case there is an apparent discrepancy between the result of Crooks and that of Jarzynski, it is worth clarifying the situation. For completeness, we also allow for the possibility where the unperturbed system is held at fixed pressure (or similar intensive parameter) rather than fixed volume.
As a simple example, to make the differences between these cases clear, we consider a gas in a container in thermal contact with a reservoir at a temperature $T$. The partition between the container and the reservoir is either at a fixed location, or free to move to maintain equal pressures in the container and the reservoir. Another wall of the container has a movable piston, which either has a definite time-varying position or has a definite force applied to it. When the reservoir has fixed volume, and the piston's position is varied in a definite manner, the Jarzynski equality is equivalent to $\langle\exp [-\beta W]\rangle=\exp [-\beta \Delta A]$, and there is no difference between $W$ and $W_{J}$ [18]. We proceed to analyze the remaining two cases.

When the reservoir has a fixed pressure, and the piston's position is varied in a definite manner, microscopic reversibility [19] in the dynamics ensures that the probability to follow a path $x(t)$ when the piston is moved along a trajectory as compared to the probability to move along the time reversed path if the piston is moved along the opposite trajectory is given by

$$
\frac{\mathcal{P}[x(+t) \mid \lambda(+t)]}{\mathcal{P}[\bar{x}(-t) \mid \bar{\lambda}(-t)]}=\exp [-\beta Q\{x(+t), \lambda(+t)\}]
$$

where $Q\{x(+t), \lambda(+t)\}$ is the heat transferred to the system from the bath along the forward trajectory, and $\lambda(t)$ denotes the variation of the external (coordinate) parameter. The notation is the same as in Ref. 11], but our result differs in the absence of a $p \Delta V$ term in the exponential on the right hand side. Eq.(6) is justified by noting that if a microstate $A$ of the system and the reservoir together evolves with time into a microstate $B$, then the time reversed microstate $B^{\prime}$ must evolve into the time reversed microstate $A^{\prime}$ when the variation of the external parameter is reversed. The right hand side of Eq.(6) is then simply the ratio of the probability of finding the reservoir in the appropriate microstate that would move the system along the forward trajectory as compared to the backward one. Using a constant pressure ensemble for the reservoir, this ratio of probabilities is equal to $\exp \left[\beta\left(U_{f}^{R}-U_{i}^{R}+p V_{f}^{R}-p V_{i}^{R}\right)\right]$, where $U_{i, f}^{R}$ and $V_{i, f}^{R}$ are the energy and volume of the reservoir in the initial and final state. (One can verify that, in the absence of any external work, such a ratio of probabilities weights states of the system in accordance with the constant pressure ensemble.) Since $\Delta U^{R}+p \Delta V^{R}=-Q$, Eq. (6) follows.

Proceeding as in Crooks [11] with the necessary changes, if the variable $\omega$ is defined as

$$
\omega=\ln p\left(x_{i}\right)-\ln p\left(x_{f}\right)-\beta Q
$$

where $p_{i, f}$ are the probabilities of the initial and final states of the system, then Eq. (5) is satisfied [20]. Using the fact that $\ln p_{i, f}=-\beta\left(U_{i, f}+p V_{i, f}\right)+\beta G_{i, f}$, where $G$ is the Gibbs free energy of the system, we obtain that $\omega=\beta(\Delta U+p \Delta V-Q-\Delta G)=\beta(W-\Delta G)$, and so

$$
\langle\exp [-\beta W]\rangle=\exp [-\beta \Delta G] .
$$


Thus the actual physical work is related to the change in the Gibbs free energy. If the process is carried out adiabatically, $W$ is always equal to $\Delta G$.

On the other hand, when the reservoir has a fixed volume but the force on the piston is varied in a definite manner, Eqs.(6), (7) and (5) still hold. However, $\ln p_{i, f}=-\beta\left(U_{i, f}+F_{i, f} x_{i, f}\right)+\beta G_{i, f}$, where $F$ and $x$ are the force exerted by the piston and its coordinate. $G=k_{B} T \ln \sum \exp [-\beta(U+F x)]$; if $F x$ is absorbed into $U$ as in [9], this would be considered as the Helmholtz free energy. Now since $\Delta U=Q-W$, where $W=\int F d x$, Eq.(15) yields

$$
\left\langle\exp \left[\beta \int x d F\right]\right\rangle=\left\langle\exp \left[-\beta W_{J}\right]\right\rangle=\exp [-\beta \Delta G]
$$

in agreement with Jarzynski's result [9].

It is also possible to consider the case when the reservoir is separated from the system by a movable barrier (that equalizes pressures on both sides) and the external force is applied to the same barrier. As expected, in this case the appropriate free energy is obtained from $U+p V+F x$, while $W_{J}=-\int x d F$. ( $V$ and $x$ are not independent variables.)

To summarize, if a coordinate of a system is varied in a specific way, the work done is related to the change in the appropriate free energy, but if a force on a system is varied, the Legendre transform of the work is related to the change in the appropriate free energy. Although the thermodynamic approach starts with the work in all cases, the final result involves the generalized work, in complete agreement with $[9]$.

To make matters clearer, we consider the experimentally relevant case [10], to which we shall return later in Section IV, in which a molecule is stretched by placing its end in a parabolic optical trap whose position is varied. If $x_{0}(t)$ is the center of the trap and $x$ is the end of the molecule, the energy in the trap is $k\left[x-x_{0}(t)\right]^{2} / 2$. If $H(x)$ is the internal energy of the molecule, the Jarzynski equality relates the change in free energy with $H(\tau)=$ $H(x)+k\left[x-x_{0}(\tau)\right]^{2} / 2$ to $W_{J}=-\int k\left[x-x_{0}(t)\right] d x_{0}$. If one considers the trap and the molecule as a single combined system, $W_{J}$ is the work done by the external agent moving the trap, and $H(t)$ is the appropriate Hamiltonian for the combined system. On the other hand, if one is only interested in the molecule, then it would be desirable to find a way to exclude the $k\left[x-x_{0}(\tau)\right]^{2} / 2$ term when computing the change in free energy. This latter option might seem more natural, and is explored in 12 . and [13]. However one could consider an alternative experiment without an optical trap, with $x$ and $x_{0}$ the positions of the second-last and last sites on the molecule, and $x_{0}$ dragged along a definite path. The calculations would then be identical, but the $k\left[x-x_{0}\right]^{2} / 2$ term - or an anharmonic generalization thereof - would be part of the stretching energy of the molecule, and $W_{J}$ would be the work done. No manipulations of the Jarzynski equality would then be sought.

\section{ALTERNATIVE DEFINITION OF ENTROPY FLUCTUATIONS}

Wang et al 7] have performed experiments where a colloidal particle in an optical trap is dragged in a solvent. The trap is initially at rest, and then from time $t=0$ to $\tau$ is dragged at a uniform velocity $v_{0}$. The instantaneous force exerted by the optical trap is $F_{\text {opt }}=-k\left(x-x_{0}\right)$, where $x$ is the position of the particle and $x_{0}$ is the position of the center of the trap. The work done by the optical trap on the particle is then equal to

$$
W(\tau)=-k \int_{0}^{\tau} d t v_{0}\left(x-x_{0}\right)
$$

An integrated form of the fluctuation theorem

$$
\frac{\operatorname{Pr}(W<0)}{\operatorname{Pr}(W>0)}=\langle\exp [-\beta W]\rangle_{W>0}
$$

is verified [7].

As discussed by Mazonka and Jarzynski [14, 15], the entropy generated can be defined as $\Delta S=W / T$ or as $\Delta S=-Q / T$, where $Q$ is the heat absorbed by the particle from the solvent, which acts as a heat reservoir. The first definition, used by Kurchan [5] and Crooks [1]], is equivalent to choosing [11] the entropy of the particle in a certain state to be $-\ln p$, where $p$ is the probability of the state, and is justified by recalling that the entropy of the particle over the entire canonical ensemble is $\langle-\ln p\rangle$. The second definition adopts the convention that the entropy of the particle for a specified position and velocity is zero, since it has no extra internal degrees of freedom, so that entropy is only generated by heat being transferred to the solvent. Both definitions are reasonable; to some extent, the entropy of a single particle in a specific configuration is arbitrary.

With $\Delta S=W / T$, it is possible to verify [14, 15] with Langevin dynamics for the particle that the TFT is satisfied, in accordance with the experimental result [7]. A corollary of this result is that the Jarzynski equality is satisfied, which in this context is $\langle\exp [-\beta W]\rangle=1$. It is also shown 14, 15] that the SSFT is valid. The question was raised [14] whether the fluctuation theorem - either transient or steady state - is satisfied with the alternative choice of $\Delta S=-Q / T$. In Ref. 15], it was shown that even in the large time limit, if $Q / \tau$ is held constant, the fluctuation theorem has to be modified for $Q$ 21]. In this section we show that, $\langle\exp [\beta Q]\rangle$, analogous to the expression for $W$ in the Jarzynski equality, diverges as $\tau \rightarrow \infty$, and also for any finite $\tau$ in the commonly used limit when the mass of the particle $m \rightarrow 0$.

Because the limit of zero particle mass is singular for $\langle\exp [\beta Q]\rangle$, we start with the Langevin equation for the particle

$$
m \ddot{x}=-\lambda \dot{x}-k\left(x-x_{0}\right)+\eta(t)
$$

where $m$ is the mass of the particle, $\lambda$ is a viscous damping coefficient, and $\eta(t)$ is the thermal noise satisfying 
$\left\langle\eta\left(t_{1}\right) \eta\left(t_{2}\right)\right\rangle=2 \lambda k_{B} T \delta\left(t_{1}-t_{2}\right)$. The center of the optical trap is specified by $x_{0}=v_{0} t \theta(t)$. The solution to Eq.(12) can be written as

$$
x(t)=\bar{x}(t)+\tilde{x}(t),
$$

where

$$
m \ddot{\bar{x}}=-\lambda \dot{\bar{x}}-k\left(\bar{x}-x_{0}\right)
$$

and

$$
m \ddot{\tilde{x}}=-\lambda \dot{\tilde{x}}-k \tilde{x}+\eta .
$$

From Eq.(10), the heat generation rate equal to

$-d Q / d t=-k v_{0}\left(x-x_{0}\right)-\frac{1}{2} \frac{d}{d t}\left[k\left(x-x_{0}\right)^{2}+m \dot{x}^{2}\right]=(\lambda \dot{x}-\eta) \dot{x}$

where Eq.(12) is used to obtain the second form. Using Eq.(13) and Eq.(15) yields the result

$$
-d Q / d t=\lambda \dot{\bar{x}}^{2}+\dot{\bar{x}}[2 \lambda \dot{\tilde{x}}-\eta]-\frac{1}{2} \frac{d}{d t}\left[k \tilde{x}^{2}+m \dot{\tilde{x}}^{2}\right] .
$$

Sufficiently long after the optical trap starts to move, $\dot{\bar{x}} \rightarrow v_{0}$. Therefore, if Eq.(17) is integrated over a sufficiently long time interval $[0, \tau]$, , it is clear that the three contributions to $-Q(\tau)$ are i) a deterministic term that approaches $\lambda v_{0}^{2} \tau$ ii) a stochastic term that is $\sim O(\sqrt{\tau})$, and iii) a random $\sim O(1)$ contribution from the total derivative. Despite the relative smallness of the third term, it is crucial in proving that the fluctuation theorem is not satisfied by $Q$ when $\tau \rightarrow \infty$ with $Q / \tau$ fixed [15, 21], because it affects the tails of the distribution where the fluctuation theorem is tested.

Even without detailed calculations, it is possible to understand the singularity of $\langle\exp [\beta Q]\rangle$ qualitatively. Both singularities come from the third term to the right hand side of Eq.(17). If $m \rightarrow 0$, $\dot{\tilde{x}}$ is uncorrelated from one instant to the next. Therefore $m\left[\dot{\tilde{x}}^{2}(0)-\dot{\tilde{x}}^{2}(\tau)\right] / 2$ decouples from the other contributions to $-Q(\tau)$. Since $\dot{\tilde{x}}(\tau)$ is drawn from the Gaussian distribution, $\sim \exp \left[-\beta m \dot{\tilde{x}}^{2}(\tau) / 2\right]$, we see that the integral over $\dot{\tilde{x}}(\tau)$ in $\langle\exp [\beta Q]\rangle$ diverges. Even when the zero mass limit is not taken, the same argument applies to $m \dot{\tilde{x}}^{2}(\tau) / 2$ and to $k \tilde{x}^{2}(\tau) / 2$ in the $t \rightarrow \infty$ limit. The full calculation is shown in the appendix.

\section{STRETCHING EXPERIMENTS ON POLYMERS}

In experiments by Liphardt et al [10], the Jarzynski equality is put to use in non-equilibrium stretching measurements on single RNA molecules, to obtain the free energy change as a result of stretching. The RNA molecule has polystyrene beads attached to the two ends. One bead (bead A) is placed in an optical trap, whose center is kept fixed throughout the experiment. The bead at the other end (bead B) is held at a definite time-dependent position using a piezoelectric actuator. The force exerted on the molecule by the optical trap is measured. If the origin of the coordinate system is taken to be at the center of the optical trap, this force is related to the position of bead A by $F=-k x$. Therefore, by measuring the force, the (fluctuating) position of bead A can be found. If $x_{0}(t)+L$ is the position of bead $\mathrm{B}$, where $L$ is the unstretched length of the molecule, the stretching is equal to $z=x_{0}-x$. The integral

$$
w=-\int F d z=k \int x d\left(x_{0}-x\right)
$$

is evaluated over a large number of trials, and the Jarzynski identity is used to obtain the change in the free energy of the molecule as a result of stretching.

Clearly, if the experiment is performed adiabatically, $w$ will always be equal to the reversible work required to stretch the molecule, i.e. the change in the free energy. However, in the irreversible case, there are two differences between $w$ and the actual work done that raise a question about the applicability of the Jarzynski identity. The first is that the force exerted on bead A, not bead $\mathrm{B}$, is measured. The second is that the stretching is measured rather than the displacement of bead B. These may seem innocuous changes; indeed, the second might even be considered desirable, since it attempts to eliminate the change in the potential energy of bead A, which is not of interest. In order to see whether these changes are indeed harmless, we consider a toy model for the system that is admittedly unrealistic: where the RNA molecule is also treated as a harmonic spring. In view of the unfolding transition seen in the molecule, this is obviously wrong. Our objective, however, is to investigate whether the difference between $w$ and the actual work done is potentially significant. In the same spirit, the damping and thermal noise forces experienced along the RNA molecule are neglected, and the mass of the beads is taken to be zero.

In this toy model, the equation for bead A is

$$
\lambda \dot{x}=-k x+\kappa\left(v_{0} t-x\right)+\eta
$$

where, as before, $k$ is the force constant of the optical trap, $\lambda$ is the viscous damping coefficient, and $\eta$ is the thermal noise. $\kappa$ is the force constant for the (harmonic version of) the RNA molecule. Bead B is assumed to be moved at constant speed $v_{0}$ over a time interval $[0, \tau]$. Proceeding as in the previous section, defining $t_{0}=\lambda /(k+\kappa)$, Eq.(19) has the solution

$$
x(t)=\frac{\kappa v_{0} t}{\kappa+k}-\frac{\kappa v_{0} t_{0}}{\kappa+k}\left(1-\exp \left[-t / t_{0}\right]\right)+\tilde{x}
$$

where

$$
\tilde{x}(t)=\tilde{x}(0) \exp \left[-t / t_{0}\right]+\int_{0}^{t} d t_{1} \frac{\eta\left(t_{1}\right)}{\lambda} \exp \left[-\left(t-t_{1}\right) / t_{0}\right] .
$$


Substituting this solution in Eq.(18) yields

$$
\begin{aligned}
d w / d t= & {\left[\frac{k \kappa v_{0}}{k+\kappa}\left\{t-t_{0}\left(1-e^{-t / t_{0}}\right)\right\}+k \tilde{x}\right] } \\
& {\left[\frac{v_{0}}{k+\kappa}\left\{k+\kappa e^{-t / t_{0}}\right\}-\dot{\tilde{x}}\right] }
\end{aligned}
$$

If $v_{0} \rightarrow 0$ while keeping $v_{0} \tau$ fixed, i.e. the adiabatic limit is taken, the only term in $w(\tau)$ that survives comes from integrating the product of the first term in each bracket. This is the change in free energy of the harmonic RNA molecule. Accordingly, the dissipative work satisfies

$$
\begin{aligned}
d w_{D} / d t & =\frac{k \kappa v_{0}^{2}}{(k+\kappa)^{2}}\left[\kappa t e^{-t / t_{0}}-t_{0}\left(k+\kappa e^{-t / t_{0}}\right)\left(1-e^{-t / t_{0}}\right)\right] \\
& +\frac{k v_{0} \tilde{x}}{k+\kappa}\left[k+\kappa e^{-t / t_{0}}\right]-\frac{k \kappa v_{0} \dot{\tilde{x}}}{k+\kappa}\left[t-t_{0}\left(1-e^{-t / t_{0}}\right)\right] \\
& -\frac{1}{2} k d \tilde{x}^{2} / d t
\end{aligned}
$$

Comparing with Eq.(17), this has the same structure of a deterministic term, a Gaussian stochastic term and a total derivative. However, if $\tau>>t_{0}$, it can be seen that the integral of the deterministic term is negative, so that $\left\langle\exp \left[-\beta w_{D}(\tau)\right]\right\rangle$ cannot be equal to 1 .

To solve for the dynamics of the system correctly, one would need to modify Eq. (19) by using the full nonlinear force from the stretching of the molecule, and including the damping and thermal noise acting along the length of the molecule. This would be difficult, and not amenable to an analytical treatment. Experimentally 10], good agreement is found between the free energy determined by non-equilibrium and adiabatic stretching. It is not clear whether this is fortuitous, or can be justified: perhaps the fact that the non-equilibrium nature of the experiments are only seen to be significant when the RNA molecule goes through its unfolding transition might make $w$ as defined in Eq. (18) better behaved. For instance, the fact that the bead in the optical trap moves backwards in the unfolding direction means that the result $\left\langle w_{D}(\tau)\right\rangle<0$ found (for $t>>t_{0}$ ) for the toy model will not be the case for the real system, so deviations from the Jarzynski equality are less dramatic.

The reliable method would be to modify the experiment and move the optical trap while keeping the other end fixed. If the force and displacement of the optical trap are measured, as in the previous section, the Jarzynski equality can be safely invoked [22]. Even with the modified experiment, the displacement of the optical trap rather than the stretching of the molecule must be used in computing the work. As discussed briefly in Section II, the Jarzynski equality will then yield the change in the free energy of the optical trap and the molecule together, i.e.

$$
\left\langle e^{-\beta w_{J}(\tau)}\right\rangle=\frac{\int d x(\tau) \exp \left[-\beta\left\{A^{\mathrm{in}}(x(\tau))+\frac{k}{2}(x(\tau)-v \tau)^{2}\right\}\right]}{\int d x(0)\left[-\beta\left\{A^{\text {in }}(x(0))+k x(0)^{2} / 2\right\}\right]}
$$

where $A^{\text {in }}$ is the internal free energy of stretching of the molecule, and $x(0), x(\tau)$ are the starting and ending positions of bead A relative to the initial center of the trap. (Bead B is fixed throughout.) On the other hand, the quantity of interest is $A^{\text {in }}$. An elegant method to obtain $A^{\text {in }}$ is due to Hummer and Szabo [12], which relies on a stronger version of the Jarzynski equality:

$\left\langle e^{-\beta w_{J}(\tau)} \delta(x(\tau)-x)\right\rangle=\frac{\exp \left[-\beta\left\{A^{\mathrm{in}}(x)+k(x-v \tau)^{2} / 2\right\}\right]}{\int d x(0)\left[-\beta\left\{A^{\mathrm{in}}(x(0))+k x(0)^{2} / 2\right\}\right]}$

where the quantity averaged on the left hand side is $\exp \left[-\beta w_{J}(\tau)\right] / \epsilon$ for experimental trials when $x<x_{\tau}<$ $x+\epsilon$ and zero otherwise, for small $\epsilon$. Summing both sides of Eq. (25) yields (24). Eq.(25) can be proved by invoking the Feynman Kac theorem [23]. The $k(x-v \tau)^{2} / 2$ term in Eq.(25) can easily be transferred to the left hand side. Numerics with different values of $\tau$, with $x$ chosen to be close to its optimal value for each $\tau$, were used [12] to obtain the free energy profile. Note that it is not correct to choose a different $\tau$ for each experimental trial so that $x(\tau)$ is always equal to $x$ and seek to apply Eq.25), i.e. to perform an experiment of variable duration with a definite extension of the molecule at the end of each trial. Such a protocol would yield

$$
\begin{aligned}
\left\langle e^{-\beta w_{J}(x)}\right\rangle & \propto \int_{0}^{\tau_{M}} d \tau \exp \left[-\beta\left\{A^{\text {in }}(x)+\frac{k}{2}(x-v \tau)^{2}\right\}\right] \\
& \propto[1+\operatorname{erf}(x \sqrt{\beta k / 2})] \exp \left[-\beta A^{\text {in }}(x)\right]
\end{aligned}
$$

if $\tau_{M} \rightarrow \infty$.

\section{FLUCTUATION THEOREMS FOR SYSTEMS WITH LANGEVIN DYNAMICS}

We consider a system of particles with generalized coordinates $x=\left\{x_{l}\right\}$ and velocities $v=\left\{v_{l}=\dot{x}_{l}\right\}$ and described by the Hamiltonian

$$
H(x, v)=\sum_{l} \frac{m_{l} v_{l}^{2}}{2}+V(x)
$$

where $V$ is an interaction potential. We assume that the system is in contact with a heat reservoir, and its time evolution is describable by Langevin dynamics. A set of external forces $\left\{f_{l}\right\}$ act on the particles and perform work on it. Thus the equations of motion are given by:

$$
m_{l} \ddot{x}_{l}=-\frac{\partial V}{\partial x_{l}}+f_{l}(t)-\lambda_{l} \dot{x}_{l}+\eta_{l}
$$

where $\eta_{l}$ is Gaussian white noise with the correlator $\left\langle\eta_{l}(t) \eta_{m}\left(t^{\prime}\right)\right\rangle=2 \lambda_{l} k_{B} T \delta\left(t-t^{\prime}\right) \delta_{l m}$. For stochastic systems, the fluctuation theorem - and therefore the Jarzynski equality - was proved by Kurchan [5]. We provide a simpler proof, through Eq.(6). For discrete systems, evolving, for example, through Monte Carlo dynamics, Eq. (6) has been proved [1]. Here we give a proof for systems evolving through Langevin dynamics. 
The principle of microscopic reversibility relates the probability of a particular path in phase space to the probability of the time-reversed path. Consider the evolution of the system from time $t=0$ to $t=\tau$ through a path specified by $\{x(t), v(t), f(t)\}$. The probability of this path is given by:

$$
\begin{aligned}
\mathcal{P}_{+} & =\mathcal{N} \exp \left[-\sum_{l} \frac{\beta}{4 \lambda_{l}} \int_{0}^{\tau} d t \eta_{l}^{2}(t)\right] \\
& =\mathcal{N} \exp \left[-\sum_{l} \frac{\beta}{4 \lambda_{l}} \int_{0}^{\tau} d t\left(m_{l} \ddot{x}_{l}+\frac{\partial V}{\partial x_{l}}-f_{l}(t)+\lambda_{l} \dot{x}_{l}\right)^{2}\right]
\end{aligned}
$$

where $\mathcal{N}$ is a normalization constant. Now consider the time-reversed path given by $\left\{\bar{x}^{\prime}(t), \bar{v}^{\prime}(t), \bar{f}^{\prime}(t)\right\}=\{\bar{x}(\tau-$ $t),-\bar{v}(\tau-t), \bar{f}(\tau-t)\}$. The probability of this path is:

$$
\begin{aligned}
\mathcal{P}_{-} & =\mathcal{N} \exp \left[-\sum_{l} \frac{\beta}{4 \lambda_{l}} \int_{0}^{\tau} d t\left(m_{l} \ddot{x}_{l}+\frac{\partial V}{\partial x_{l}^{\prime}}-f_{l}^{\prime}(t)+\lambda_{l} \dot{x}_{l}^{\prime}\right)^{2}\right] \\
& =\mathcal{N} \exp \left[-\sum_{l} \frac{\beta}{4 \lambda_{l}} \int_{0}^{\tau} d t\left(m_{l} \ddot{x}_{l}+\frac{\partial V}{\partial x_{l}}-f_{l}(t)-\lambda_{l} \dot{x}_{l}\right)^{2}\right]
\end{aligned}
$$

Taking the ratio of the two probabilities leads to the principle of microscopic reversibility

$$
\begin{aligned}
\frac{\mathcal{P}_{+}}{\mathcal{P}_{-}} & =\exp \left[-\beta \sum_{l} \int_{0}^{\tau} d t\left[m_{l} \ddot{x}_{l}+\frac{\partial V}{\partial x_{l}}-f_{l}(t)\right] \dot{x}_{l}\right] \\
& =\exp [-\beta Q]
\end{aligned}
$$

where $Q$ is the amount of heat transferred from the heat bath to the system. The identification of $Q$ as the heat transfer can be seen either by noting that $Q=\sum_{l} \int_{0}^{\tau}\left[m_{l} \ddot{x}_{l}+\frac{\partial V}{\partial x_{l}}-f_{l}(t)\right] \dot{x}_{l}=\sum_{l} \int_{0}^{\tau} d t\left[-\lambda_{l} \dot{x}_{l}+\eta_{l}\right] \dot{x}_{l}$ which is clearly the energy flow from the heat bath. Note that in the absence of external forces the principle of microscopic reversibility reduces to the usual detailed balance principle which states that:

$$
\frac{P\left(x_{f}, v_{f} \mid x_{i}, v_{i}\right)}{P\left(x_{i},-v_{i} \mid x_{f},-v_{f}\right)}=e^{-\beta\left[H\left(x_{f}, v_{f}\right)-H\left(x_{i}, v_{i}\right)\right]}
$$

where $P\left(x, v \mid x^{\prime}, v^{\prime}\right)$ denotes the probability of being at $(x, v)$ at time $t=\tau$ given that it was at $\left(x^{\prime}, v^{\prime}\right)$ at time $t=0$. This follows if we integrate Eq. (31) over all paths between $\left(x_{i}, v_{i}\right)$ and $\left(x_{f}, v_{f}\right)$. From Eqs. (31) and (32), the fluctuation theorem and the Jarzynski equality can be obtained as in Crooks 11], discussed in Section II. In particular, when the external forces are switched on at time $t=0$,

$$
\begin{aligned}
& \left\langle\exp \left[\beta \int_{0}^{\tau} x \cdot d f\right]\right\rangle=\left\langle\exp \left[\beta f(\tau) \cdot x(\tau)-\beta \int_{0}^{\tau} d t f \cdot v\right]\right\rangle \\
= & \frac{\langle\exp [-\beta H(x(\tau), v(\tau))+\beta f(\tau) \cdot x(\tau)]\rangle_{x(\tau), v(\tau)}}{\langle\exp [-\beta H(x(0), v(0))]\rangle_{x(0), v(0)}} .
\end{aligned}
$$

As discussed after Eq.(25), Eq.(33) is true even without $x(\tau)$ being averaged, so that $\exp [\beta f(\tau) \cdot x(\tau)]$ can be canceled on both sides and

$$
\left\langle\exp \left[-\beta \int_{0}^{\tau} d t f \cdot v\right]\right\rangle=1 .
$$

If one compares the system starting in thermal equilibrium with a force $f(t)$ switched on for $0<t<\tau$, with the same system but with a force $f(\tau-t)$, then the variable $\omega$ in Ref. [1] is

$$
\begin{aligned}
\omega= & \ln \rho\left(x_{i}, v_{i}\right)-\ln \rho\left(x_{f}, v_{f}\right)-\beta Q \\
= & \ln \rho\left(x_{i}, v_{i}\right)-\ln \rho\left(x_{f}, v_{f}\right) \\
& -\beta\left[H\left(x_{f}, v_{f}\right)-H\left(x_{i}, v_{i}\right)-\int_{0}^{\tau} d t f \cdot v\right] \\
= & \beta \int_{0}^{\tau} d t f \cdot v .
\end{aligned}
$$

When the force that is switched on is time independent, then Eq.(4) reduces to the result of Ref. [5]. In fact, even when $f(t)$ depends on time, if the equations of motion Eq. (28) are linear, the distribution of $\omega=\beta \int_{0}^{\tau} d t f \cdot v$ is Gaussian for both the original and the time reversed process, i.e.

$$
\frac{p_{+}(\omega)}{p_{-}(-\omega)} \propto \frac{\exp \left[-\left(\omega-m_{+}\right)^{2} / 2 \sigma_{+}\right]}{\exp \left[-\left(\omega+m_{-}\right)^{2} / 2 \sigma_{-}\right]} .
$$

Comparing to Eq.(4) we see that $\sigma_{+}=\sigma_{-}$and $m_{+}^{2} / \sigma_{+}=$ $m_{-}^{2} / \sigma_{-}$, i.e. $m_{+}=m_{-}$(since $\left.m_{ \pm}>0\right)$. Further, $m_{ \pm} / \sigma_{ \pm}=\frac{1}{2}$. One can thus drop the subscripts in Eq.(4) for linear equations of motion for arbitrary $f(t)$.

More generally, when the interaction potential depends on a set of externally controlled parameters $\mu(t)$ so that we have $H=H(x, v, \mu)$. In this case we get

$$
\begin{aligned}
Q & =\sum_{l} \int_{0}^{\tau}\left[m_{l} \ddot{x}_{l}+\frac{\partial V(x, \mu)}{\partial x_{l}}\right] \dot{x}_{l} \\
& =\int_{0}^{\tau} d t\left[\frac{d}{d t}\left(\sum_{l} \frac{m_{l} v_{l}^{2}}{2}+V\right)-\sum_{l} \frac{\partial V}{\partial \mu_{l}} \dot{\mu}_{l}\right] \\
& =H\left(x_{f}, v_{f}, \mu_{f}\right)-H\left(x_{i}, v_{i}, \mu_{i}\right)-W_{J}
\end{aligned}
$$

Now if we take $\rho=e^{-\beta[H(x, v, \mu)-F(\mu)]}$ where $F(\mu)=$ $-(1 / \beta) \ln \left(\int d x d v e^{-\beta H(x, v, \mu)}\right)$ is a generalized free energy then we get the Jarzynski result:

$$
\left\langle e^{-\beta W_{J}}\right\rangle=e^{-\beta\left[F\left(\mu_{f}\right)-F\left(\mu_{i}\right)\right]}
$$

The principle of microscopic reversibility can be used to derive some other interesting results that are related to the Jarzynski result and have been derived using other approaches. We again consider the case with a time dependent Hamiltonian in which case Eq. (31) gives $e^{-\beta W_{J}} \mathcal{P}_{+}=e^{-\beta\left[H\left(x_{f}, v_{f}, \mu_{f}\right)-H\left(x_{i}, v_{i}, \mu_{i}\right)\right]} \mathcal{P}_{-}$. Integrating both sides over all paths between the fixed initial and final points then gives:

$$
\begin{aligned}
& \left\langle e^{-\beta W_{J}}\right\rangle_{\left(x_{i}, v_{i}\right)}^{\left(x_{f}, v_{f}\right)} \\
= & P_{R}\left(x_{i},-v_{i} \mid x_{f},-v_{f}\right) e^{-\beta\left[H\left(x_{f}, v_{f}, \mu_{f}\right)-H\left(x_{i}, v_{i}, \mu_{i}\right)\right.}(
\end{aligned}
$$


where $P_{R}\left(x_{i},-v_{i} \mid x_{f},-v_{f}\right)$ is the transition probability under the action of the time reversed Hamiltonian. If we now integrate over all initial states chosen from the canonical ensemble, Eq.(25) results.

\section{CONCLUSION}

In this paper, we have analyzed recent experimental tests of the Fluctuation Theorem and the Jarzynski equality. For a particle dragged through a solvent [3], the heat absorbed, which has been suggested as an alternative definition [14, 15] of the entropy generated, violates the Jarzynski equality. In the experiments on molecular stretching [10], the fact that the force is not measured at the end of the molecule that is dragged can lead to substantial deviations from the Jarzynski equality, even changing the sign of the average 'work'. We have also provided a proof of the fluctuation theorem for systems governed by Langevin dynamics that is much simpler than the standard proof 5], and have generalized it slightly for linear Langevin equations. Finally, we have also clarified the connection between the thermodynamic work used in a thermodynamic derivation of the Jarzynski equality 11] and the generalized work in the original derivation [9], verifying that Eq. (3) is obtained in all cases.

\section{Acknowledgments}

It is a pleasure to acknowledge useful discussions with Jan Liphardt, Joshua Deutsch and Narayanan Menon. We also thank Michael Schurr and Narayanan Menon for providing us with copies of their papers prior to release. AD acknowledges support from the NSF under grant DMR 0086287.

\section{APPENDIX A: GAUSSIAN INTEGRAL}

In this appendix, we evaluate the singularity in $\langle\exp [\beta Q]\rangle$ discussed in Section III. Defining Gaussian variables

$$
\begin{aligned}
y_{1} & =\int_{0}^{\tau} d t \dot{\bar{x}}[2 \lambda \dot{\tilde{x}}(t)-\eta(t)] \\
y_{2} & =\sqrt{m} \dot{\tilde{x}}(\tau) \\
y_{3} & =\sqrt{m} \dot{\tilde{x}}(0) \\
y_{4} & =\sqrt{k} \tilde{x}(\tau) \\
y_{5} & =\sqrt{k} \tilde{x}(0)
\end{aligned}
$$

we have

$$
\beta Q(\tau)=-\beta \lambda \int_{0}^{\tau} \dot{\bar{x}}^{2}-\beta y_{1}+\frac{1}{2} \beta\left[y_{2}^{2}-y_{3}^{2}+y_{4}^{2}-y_{5}^{2}\right]
$$

From Eq.(14), it is straightforward to see that

$$
\bar{x}(t)=v_{0} t-\frac{\lambda v_{0}}{k}+A_{1} \exp \left[-\alpha_{1} t\right]+A_{2} \exp \left[-\alpha_{2} t\right]
$$

where the two exponentially decaying terms are the solutions to the homogeneous equation for $x$ from Eq.(12), satisfying

$$
\alpha_{1} \alpha_{2}=\frac{k}{m}, \quad \alpha_{1}+\alpha_{2}=\frac{\lambda}{m} .
$$

With the initial condition $\bar{x}(0)=\dot{\bar{x}}(0)=0$,

$$
A_{1,2}=\frac{v_{0}\left[1-\lambda \alpha_{2,1} / k\right]}{\alpha_{1,2}-\alpha_{2,1}}
$$

Similarly, from Eq.(15), one obtains

$$
\begin{aligned}
\tilde{x}(t) & =\frac{\alpha_{1} \tilde{x}(0)+\dot{\tilde{x}}(0)}{\alpha_{1}-\alpha_{2}} e^{-\alpha_{2} t}+\frac{\alpha_{2} \tilde{x}(0)+\dot{\tilde{x}}(0)}{\alpha_{2}-\alpha_{1}} e^{-\alpha_{1} t} \\
& +\int_{0}^{t} d t^{\prime} \frac{\eta\left(t^{\prime}\right)}{m} \frac{e^{-\alpha_{1}\left(t-t^{\prime}\right)}-e^{-\alpha_{2}\left(t-t^{\prime}\right)}}{\alpha_{2}-\alpha_{1}}
\end{aligned}
$$

Using Eq. (A6), it is possible to obtain the correlation between the Gaussian variables $y_{1} \ldots y_{5}$, as follows:

$$
\begin{aligned}
\left\langle y_{1}^{2}\right\rangle & =2 \lambda k_{B} T \int d t \dot{\bar{x}}^{2}(t) \\
\left\langle y_{1} y_{2}\right\rangle & =\left\langle y_{1} y_{4}\right\rangle=0 \\
\left\langle y_{1} y_{3}\right\rangle & =\frac{2 \lambda k_{B} T}{\sqrt{m}} \int d t \dot{\bar{x}}(t) \frac{\alpha_{2} e^{-\alpha_{2} t}-\alpha_{1} e^{-\alpha_{1} t}}{\alpha_{2}-\alpha_{1}} \\
\left\langle y_{1} y_{5}\right\rangle & =\frac{2 \lambda k_{B} T \sqrt{k}}{m} \int d t \dot{\bar{x}}(t) \frac{e^{-\alpha_{2} t}-e^{-\alpha_{1} t}}{\alpha_{2}-\alpha_{1}} \\
\left\langle y_{2}^{2}\right\rangle & =\left\langle y_{3}^{2}\right\rangle=\left\langle y_{4}^{2}\right\rangle=\left\langle y_{5}^{2}\right\rangle=k_{B} T \\
\left\langle y_{2} y_{4}\right\rangle & =\left\langle y_{3} y_{5}\right\rangle=0 \\
\left\langle y_{3} y_{4}\right\rangle & =-\left\langle y_{2} y_{5}\right\rangle=\sqrt{\frac{k}{m}} k_{B} T \frac{e^{-\alpha_{1} \tau}-e^{-\alpha_{2} \tau}}{\alpha_{2}-\alpha_{1}} \\
\left\langle y_{2} y_{3}\right\rangle & =\sqrt{\frac{m}{k}} \frac{d}{d \tau}\left\langle y_{3} y_{4}\right\rangle \\
\left\langle y_{4} y_{5}\right\rangle & =k_{B} T \frac{\alpha_{2} e^{-\alpha_{1} \tau}-\alpha_{1} e^{-\alpha_{2} \tau}}{\alpha_{2}-\alpha_{1}}
\end{aligned}
$$

In terms of the correlation matrix $M_{i j}=\left\langle y_{i} y_{j}\right\rangle$,

$$
\begin{aligned}
\langle\exp [\beta Q]\rangle & \propto \int d y_{1} \ldots d y_{5} \exp \left[-\beta \lambda \int \dot{\bar{x}}^{2}-\beta y_{1}\right. \\
+ & \left.\frac{1}{2} \beta\left(y_{2}^{2}-y_{3}^{2}+y_{4}^{2}-y_{5}^{2}\right)-\frac{1}{2} \sum_{i j} y_{i} M_{i j}^{-1} y_{j}\right]
\end{aligned}
$$

where the normalization is obtained performing the same integral with all terms in the exponential dropped except for the last. The integral is formally straightforward: defining the matrix

$$
N=M^{-1}+\beta \operatorname{diag}(0,-1,1,-1,1)
$$

one obtains

$$
\langle\exp [\beta Q]\rangle=\exp \left[-\beta \lambda \int \dot{\bar{x}}^{2}+\frac{1}{2} \beta^{2} N_{11}^{-1}\right] / \sqrt{\operatorname{det} N \operatorname{det} M} .
$$

To see the singularity clearly we first take the $m \rightarrow 0$ limit. From Eq. A4), one of $\alpha_{1,2}$ must diverge in this 
limit; we choose this to be $\alpha_{2}$. Dropping all terms with $\exp \left[-\alpha_{2} \tau\right]$ in the correlation matrix $M$, and defining $\epsilon=$ $\alpha_{1}(m / k)^{1 / 2}=(k / m)^{1 / 2} / \alpha_{2}$,

$$
\frac{M}{k_{B} T}=\left(\begin{array}{ccccc}
B(\tau) & 0 & \epsilon C(\tau) & 0 & C(\tau) \\
0 & 1 & -\epsilon^{2} E(\tau) & 0 & -\epsilon E(\tau) \\
\epsilon C(\tau) & -\epsilon^{2} E(\tau) & 1 & \epsilon E(\tau) & 0 \\
0 & 0 & \epsilon E(\tau) & 1 & E(\tau) \\
C(\tau) & -\epsilon E(\tau) & 0 & E(\tau) & 1
\end{array}\right)
$$

where

$$
\begin{aligned}
B(\tau) & =2 \lambda \int d t \dot{\bar{x}}^{2}(t) \\
C(\tau) & =-\frac{2 \lambda \sqrt{k}}{m\left(\alpha_{2}-\alpha_{1}\right)} \int d t \dot{\bar{x}}(t) \exp \left[-\alpha_{1} t\right] \\
E(\tau) & =\frac{\alpha_{2}}{\alpha_{2}-\alpha_{1}} \exp \left[-\alpha_{1} \tau\right] .
\end{aligned}
$$

Notice that $B(\tau), C(\tau), E(\tau)$ are not singular as $m \rightarrow 0$. Changing variables from $y_{2}, y_{4}$ to $\left(y_{2}+\epsilon y_{4}\right) /\left(1+\epsilon^{2}\right)^{1 / 2}$ and $\left(y_{4}-\epsilon y_{2}\right) /\left(1+\epsilon^{2}\right)^{1 / 2}$, the matrix $M / k_{B} T$ changes to

$$
\left(\begin{array}{ccccc}
B(\tau) & 0 & \epsilon C(\tau) & 0 & C(\tau) \\
0 & 1 & 0 & 0 & 0 \\
\epsilon C(\tau) & 0 & 1 & \epsilon \sqrt{1+\epsilon^{2}} E(\tau) & 0 \\
0 & 0 & \epsilon \sqrt{1+\epsilon^{2}} E(\tau) & 1 & \sqrt{1+\epsilon^{2}} E(\tau) \\
C(\tau) & 0 & 0 & \sqrt{1+\epsilon^{2}} E(\tau) & 1
\end{array}\right)
$$

The second row and column decouple from everything else. From Eq. A9, the second row and column of the matrix $N$ are then zero. (The diagonal matrix in Eq. (A9) is not affected by the change of variables from Eq. A11 to Eq.A13).) Taking the $m \rightarrow 0$ limit in all other rows and columns of $M$, it is possible to see that this is the only singularity in this limit in Eq. A10.

When $m$ is very small but not zero, it is easy to see that the offdiagonal terms $M_{2 i}=M_{i 2}$ are $O\left(\exp \left[-\alpha_{2} \tau\right]\right)$, while $M_{22}$ is still $k_{B} T$. Therefore $N_{2 i}=N_{i 2}$ are $O\left(\exp \left[-\alpha_{2} \tau\right]\right)$ and $N_{22}$ is $O\left(\exp \left[-2 \alpha_{2} \tau\right]\right)$. In Eq.(A10), $\operatorname{det} M \operatorname{det} N$ is $O\left(\exp \left[-2 \alpha_{2} \tau\right]\right)$, while $N_{11}^{-1}$ is regular as $m \rightarrow 0$. Therefore

$$
\langle\exp [\beta Q]\rangle \sim O\left(\exp \left[\alpha_{2} \tau\right]\right) \sim O(\exp [\lambda \tau / m])
$$

where $\alpha_{2}=\left[\lambda+\left(\lambda^{2}-4 k m\right)^{1 / 2}\right] /(2 m) \approx \lambda / m$ has been used.

Even when $m$ is not small, in the $\tau \rightarrow \infty$ limit, $\exp \left[-\alpha_{1,2} \tau\right] \rightarrow 0$. In Eqs. (A7), in this limit all crosscorrelations are zero except for $\left\langle y_{1} y_{3}\right\rangle$ and $\left\langle y_{1} y_{5}\right\rangle$. Thus the second and fourth rows and columns of the matrix $N$ are zero in this limit. More accurately, since $\exp \left[-\alpha_{2} \tau\right]<<\exp \left[-\alpha_{1} \tau\right]$ one can proceed as above (without assuming that $\epsilon$ is small), and obtain that $N_{2 i}=N_{i 2}=O\left(\exp \left[-\alpha_{2} \tau\right]\right)$ and $N_{22}=O\left(\exp \left[-2 \alpha_{2} \tau\right]\right)$ as before. In addition, in the remaining $4 \times 4$ submatrix formed by eliminating the second row and column, $N_{4 i}=N_{i 4}=O\left(\exp \left[-\alpha_{1} \tau\right]\right)$ and $N_{44}=O\left(\exp \left[-2 \alpha_{1} \tau\right]\right)$. Therefore

$$
\langle\exp [\beta Q]\rangle \sim O\left(\exp \left[\left(\alpha_{2}+\alpha_{1}\right) \tau\right]\right) \sim O(\exp [\lambda t / m])
$$

This has the same form as the previous equation, though the singularity in the two cases come from the $m \rightarrow 0$ and the $\tau \rightarrow \infty$ limit respectively.
[1] G. Gallavotti and E.G.D. Cohen, Phys. Rev. Lett. 74, 2694 (1995).

[2] D.J. Evans, E.G.D. Cohen and G.P. Morriss, Phys. Rev. Lett. 712401 (1993).

[3] D.J. Evans and D.J. Searles, Phys. Rev. E50, 1645 (1994).

[4] R. van Zon and E.G.D. Cohen, cond-mat/0212311

[5] J. Kurchan, J. Phys. A 31, 3719 (1998).

[6] F. Bonetto, G. Gallavotti and P.L. Garrido, Physica D 105, 226 (1997); S. Aumaitre, S. Fauve, S. McNamara and P. Poggi, Eur. Phys. J. B19, 449 (2001); D.J. Searles and D.J. Evans, J. Chem. Phys. 113, 3503 (2000); D.J. Evans, D.J. Searles and E. Mittag, Phys. Rev. E65, 051105 (2001); G. Ayton, D.J. Evans and D.J. Searles, J. Chem Phys. 115, 2033 (2001).

[7] G.M. Wang, E.M. Sevick, E. Mittag, D.J. Searles and D.J. Evans, Phys. Rev. Lett. 89, 050601 (2002).

[8] S. Ciliberto and C. Laroche, J. Phys. IV 8, 215 (1998); K. Feitosa and N. Menon, preprint.

[9] C. Jarzynski, Phys. Rev. Lett. 78, 2690 (1997).

[10] J. Liphardt, S. Dumont, S.B. Smith, I. Tinoco and C. Bustamante, Science 296, 1832 (2002).

[11] g. Crooks, Phye. Rev. E60, 2721 (1999).
[12] G. Hummer and A. Sazbo, Proc. Nat. Acad. Sci. 98, 3658 (2001).

[13] M. Schurr and B.S. Fujimoto, preprint.

[14] O. Mazonka and C. Jarzynski, cond-mat/9912121

[15] R. van Zon and E.G.D. Cohen, Phys. Rev. Lett. (in press) and cond-mat/0305147

[16] Strictly speaking, in the case of Langevin dynamics, the force exerted on the end of the molecule by the external agent differs from the stretching force by the viscous drag and thermal noise terms acting on the end. However, recognizing that these terms ultimately arise from Hamiltonian interactions with the reservoir, $W$ is equal to $W_{J}$.

[17] The imposition of a specified coordinate can be done through a hard constraint rather than a potential. For instance, in the case of a gas confined to a container, the Hamiltonian does not change when the container expands, but the range over which the coordinates can vary does. However, this can be understood as the singular limit of a potential that is zero inside the container and grows extremely rapidly outside the container. One can verify that the force exerted on the wall is equal to the derivative of the wall-system potential (and therefore the 
Hamiltonian) with respect to the wall coordinate, so that $W=W_{J}$.

[18] Even in this case, $\Delta A$ is not the change in the Helmholtz free energy of the system and the reservoir together, but the change that would have occurred if their temperature had been held constant while the system Hamiltonian was varied. (Under such a hypothetical change, the free energy of the reservoir is unchanged, so that the change in free energy of the system and the reservoir together is equal to the change in free energy of the system.) In reality, the work done by the external agent changes the temperature slightly. Though for a sufficiently large reservoir this temperature change is negligible, it still leads to a finite change in the free energy of the reservoir: $\Delta A($ res $)=-S($ res $) \Delta T=\left[S(\right.$ res $) / C_{v}($ res $\left.)\right] \Delta Q$. We thank Joshua Deutsch for discussions that helped clarify this point.

[19] Microscopic reversibility is the requirement that when the velocities of all degrees of freedom are reversed, including those of the reservoir, the dynamics are reversed.
This includes dissipative systems, as long as there are no velocity-dependent forces. The improbability of such a reversal is given by Eq. (6).

[20] Crooks considers $k_{B} \omega$ to be the entropy generated, a point that is discussed further in the next Section of this paper. For the purpose of deriving the Jarzynski equality, it does not matter what $\omega$ is.

[21] In an earlier version of this paper, it was claimed that the TFT for $Q$ is valid in the large time limit for this experiment. The error in this was pointed out by R. van Zon and E.G.D. Cohen in cond-mat/0307297

[22] One might be tempted to modify Eq.(18) as $d w=k x d x_{0}$ and argue that this is equivalent to moving the optical trap from Galilean invariance. However, this neglects the damping from the solvent, and can (with our toy model) be verified to be incorrect.

[23] Z. Schuss, Theory and applications of Stochastic Differential Equations (Wiley, New York, 1980). 